\title{
III. Ein Wort über das Symmetriecentrum.
}

\author{
Von
}

F. Becke in Prag.

Vielleicht sollte ich die folgenden Zeilen betiteln: Ein Wort fur das Symmetrie-Centrum. Veranlassung zu denselben ist die Behandlung, die dieser einfache Begriff in dem Lehrbuche der physikalischen Krystallographie von P. Groth 3. Auflage erfabren hat. In diesem vortrefflichen Buche wird zum ersten Male der Versuch gemacht, einem uber den engsten Kreis der zunftigen Krystallographen hinausgehenden Leserpublikum eine vollständige Uebersicht der 32 Symmetrieabtheilungen der Krystalle zu geben. Die Ableitung derselben erfolgt mit Hulfe der Begriffe Symmetrie-Axe, Symmetrie-Ebene und der Combination beider, der Axe und Ebene der zusammengesetzten Symmetrie. Vom Symmetrie-Centrum wird gesagt (Seite 320) : „Dasselbe ist also kein besonderes Symmetrie-Element der Krystalle, sondern ein specieller Fall der einfachen oder zusammengesetzten Symmetrie nach einer Ebene, was Fed or ow zuerst klar hervorgehoben hat. "

Dieser Satz erschien mir nicht einwurfsfrei, und ich glaube jeder, der die klare und anschauliche Darlegung des Symmetriebegriffes in der klassischen Abhandlung von Bravais uber die symmetrischen Polyëder kennt ${ }^{*}$ ), wird sich schwer uberzeugen lassen, dass zwischen den Begriffen Symmetrie-Centrum und Symmetrie-Ebene ein solcher Unterschied in der Bewerthung berechtigt sei.

Es ist meine Absicht zu zeigen, dass Symmetrie-Ebene und SymmetrieCentrum vollkommen gleichberechtigte Begriffe sind, und dass eine luckenlose Ableitung der 32 Krystallklassen möglich ist, indem man sich blos auf die Begriffe Symmetrie-Axe und Symmetrie-Centrum stutzt, wobei dann im Resultate in gewissen Abtheilungen Symmetrie-Ebenen als Folge sich einstellen, gerade so wie das in der Groth'schen Ableitung mit dem Symmetrie-Centrum der Fall ist.

*) Durch die Ueberselzung von C. und E. Blasius in Ostwald's Klassikern der exacten Wissenschaften Nr. 17 allgemein zugänglich. 
Ich knüpfe unmittelbar an die im Groth'schen Buche auf Seile 311 gegebene Darlegung des Begriffes Symmetrie an: „Als Symmelrie einer Krystallform bozeichnet man diejenige Regelmässigkeit, welche durch die Existenz gleichwerlhiger Flächen an derselben bedingt wird." "Bei krystallographischen Polyëdern sind nur gewisse Arten der Symmetrie möglich. Um diese zu erhalten, denken wir uns an einem derartig beschaffenen Krystalle die je zweien gleich werthigen Wachsthumsrichtungen entsprechenden Flächen gleichweit vom Ursprunge jener Richtungen. Wenn durch irgend eine Operation die eine der beiden Wachsthumsrichtungen so zur Deckung mit der anderen gebracht wird, dass ihre Anfangspunkte und ihre Richtungen coincidiren, dann werden offenbar auch die beiden gleichwerthigen Ebenen zusammenfallen."

In der Aufzahlung dieser Operationen weiche ich nun von Groth's Darlegungen ab wie folgl: Die Operationen können dreierlei sein:

a) Eine Drehung um eine Axe (vgl. Groth, S. 312). Sie fuhrt zum Begriff Symmetrieaxe.

b) Die Inversion. Dies ist der Fall, wenn jede uber den Ursprung binaus nach der entgegengesetzlen Seite verlangerte Richtung eine gleichwerthige Richtung darstellt; ein Polyëder mit derartiger Symmetrie heisst centrisch symmetrisch, es besitzl ein Symmetriecentrum.

c) Eine Drehung um eine Axe, verbunden mit einer Inversion. Diese Combination der beiden ersten Operationen heisse eine Inversionsdreh ung, und von einem Polyëder, welches durch eine Inversionsdrehung mil sich selbst zur Deckung gebracht wird, sagt man, es besitze eine Inversionsaxe.

Entsprechend der Ordnungszahl der zu Grunde liegenden Drehung kann die Inversionsaxe zwei-, drei-, vier-oder sechszäblig sein.

Der Begriff Inversionsaxe ist dem der Axe und Ebene der zusammengesetzlen Symmetrie vollkommen analog und zwischen beiden und den einfachen Symmetrie-Elementen besteben folgende Beziehungen. Dabei werden in Anlehnung an Brava is einige Symbole gebraucht:

$C=$ Symmetriecentrum.

$L^{n}=n$-zählige Symmetrieaxe.

$P=$ Symmetrie-Ebene.

$J^{n}=n$-zählige Inversionsaxe.

$Z^{n}=n$-zählige Axe und Ebene der zusammengesetzten Symmetrie.

$\begin{array}{ll}Z^{2}=C & J^{2}=P \\ Z^{3}=L^{3} P & J^{3}=L^{3} C \\ Z^{4}= & J^{4} \\ Z^{6}=L^{3} C & J^{6}=L^{3} P .\end{array}$

Es ist also ferner: $\quad Z^{3}=J^{6}$ und $Z^{6}=J^{3}$. 
Die Abtheilung der 32 Klassen der Krystalle gestaltet sich nun mit Hulfe der drei Begriffe: Symmetrieaxe, Symmetriecentrum und Inversionsaxe folgendermissen.

Jene Klassen von Krystallen, welche lediglich Symmetrieaxen besitzen, werden in derselben Weise abgeleilet wie bei Groth. Man erbält somit:

1. Krystalle ohne Symmetrie (Gr. 1)*). Beispiel: Saures weinsaures Strontium.

a) Krystalle mil einer einzigen Symmetrieaxe :

2. Krystalle mit einer zweizähligen Symmetrieaxe (Gr. 3). Symbol: $L^{2}$. Rohrzucker.

3. Krystalle mit einer dreizähligen Symmetrieaxe (Gr. 16). Symbol: $L^{3}$. Jodsuccinit.

4. Krystalle mit einer vierzähligen Symmetrieaxe (Gr. 10). Symbol: $L^{4}$. Wulfenit.

5. Krystalle mit einer sechszäbligen Symmetrieaxe (Gr. 23). Symbo': $L^{6}$. Nephelin.

$\beta$ ) Krystalle mit mehreren Symmetrieaxen, von denen eine senkrecht steht auf der Ebene der ubrigen:

6. Krystalle mit drei sich rech(winkelig kreuzenden, zweizähligen Symmetrieaxen (Gr. 6). Symbol: $L^{2} \cdot L_{1}{ }^{2} \cdot L_{11}{ }^{2}$. Billersaly.

7. Krystalle mit einer dreizăhligen und drei zweizähligen Symmetrieaxen, die auf der dreizähligen senkrecht stehen (Gr. 18). Symbol : $L^{3} \cdot 3 L^{2}$. Quarz.

8. Krystalle mit einer vierzähligen und zweimal zwei zweizähligen Symmetrieaxen, die auf der vierzähligen senkrecht stehen (Gr. 12). Symbol: $L^{4} .2 L^{2} .2 L_{1}{ }^{2}$. Chininsulfat.

9. Krystalle mit einer sechszäbligen und senkrecht zu derselben zweimal drei zweizähligen Symmetrieaxen (Gr. 24). Symbol: $L^{6} \cdot 3 L^{2} \cdot 3 L_{1}{ }^{2}$. Weinsaures Antimonyl-Baryum + salpetersaures Kalium.

y) Krystalle mit gleichen Symmetrieaxen, die nicht in einer Ebene liegen.

10. Krystalle mit vier dreizähligen Symmetrieaxen orientirt nacb den Ecken des Wüfels und drei zweizähligen parallel den Kanteu des Würfels (Gr. 28). Symbol: $4 L^{3} \cdot 3 L^{2}$. Natriumchlorat.

11. Krystalle mit vier dreizähligen Symmetrieaxen orientirt nach den Ecken des Würfels, drei vierzähligen parallel den Wüfelkanten und sechs zweizähligen, die die Winkel der vierzähligen balbiren (Gr. 29). Symbol: $4 L^{3} .3 L^{4} .6 L^{2}$. Salmiak.

*) In dieser Weise wird die Nummer der bstreffenden Klasse im Groth'sche. Buche angezeigt. 
Soweit stimmt also die Ableitung mit der Groth'schen ủberein. Führt man nun das Symmetriecentrum ein, indem man dasselbe dem Symbol jeder der vorstehenden 11 Klassen hinzufügt, so erhält man folgende 11 neue Klassen :

12. Krystalle mit einem Symmetriecentrum (Gr. 2). Symbol: $C$. Axinit.

13. Krystalle mit einer zweizăhligen Symmetrieaxe und einem Symmetriecentrum (Gr. 5). Symbol: $L^{2} . C$. Gyps.

14. Krystalle mit einer dreizähligen Symmetrieaxe und einem Symmetriecentrum (Gl. 17). Symbol: $L^{3} . C$. Dolomit.

15. Krystalle mit einer vierzähligen Symmetrieaxe und einem Symmetriecentrum (Gr. 13). Symbol: $L^{4} . C$. Scheelit.

16. Krystalle mit einer sechszähligen Symmetrieaxe und einem Symmetriecentrum (Gr. 25). Symbol : $L^{6} . C$. Apatit.

17. Krystalle mit drei senkrecht zu einander orientirten, zweizähligen Symmetrieaxen und einem Symmetriecentrum (Gr. 8). Symbol: $L^{2} \cdot L_{1}{ }^{2} \cdot L_{11}{ }^{2}$.C. Baryt.

18. Krystalle mit einer dreizäbligen, senkrecht dazu drei zweizähligen Symmetrieaxen und einem Symmetriecentrum (Gr. 21). Symbol: $L^{3} \cdot 3 L^{2} . C$. Calcit.

19. Krystalle mit einer vierzähligen, senkrecht dazu zweimal zwei zweizähligen Symmetrieaxen und einem Symmetriecentrum(Gr.15). Symbol: $L^{4} .2 L_{1}{ }^{2} .2 L_{11}{ }^{2}$.C. Zinnstein.

20. Krystalle mit einer sechszähligen, senkrecht dazu zweimal drei zweizähligen Symmetrieaxen und einem Symmelriecentrum (Gr. 27). Symbol: $L^{6} .3 L_{1}^{2}, 3 L_{11}{ }^{2} . C$. Beryll.

21. Krystalle mit vier dreizähligen Symmetrieaxen gerichtet nach den Würfelecken, drei zweizähligen Symmetrieaxen parallel den Würfelkanten und einem Symmetriecentrum (Gr.30). Symbol: $4 L^{3} .3 L^{2}$.C. Pyrit.

22. Krystalle mit vier dreizähligen Symmetrieaxen gerichtet nach den Würfelecken, drei vierzăhligen parallel den Würfelkanten und sechs zweizähligen, welche die Winkel der vierzähligen balbiren und einem Symmetriecentrum (Gr. 32). Symbol: $4 L^{3} \cdot 3 L^{4} \cdot 6 L^{2} . C$. Fluorit.

Aus den bei Klasse 11-22 angegebenen Symbolen lassen sich leicht die hier vorkommenden Symmetrie-Ebenen ableiten, indem man berucksichtigt, dass an centrisch-symmetrischen Krystallen senkrecht zu jeder geradzähligen Symmetrieaxe eine Symmetrie-Ebene vorhanden ist.

Nachdem so die einfachen Symmetrie-Elemente und deren mögliche Combinationen betrachtet sind, gelangen wir zu den Klassen, denen bloss Inversionsaxen zukommen. Wir betrachten zunächst den Fall einer einzigen Inversionsaxe, welche entweder zwei-, drei-, vier- oder sechszählig sein kann. Dabei stellt sich aber beraus, dass der Fall $J^{3}$ bereits durch die Abtheilung 14 dargestellt wird, indem $J^{3}=L^{3} C$. Somit bleiben ubrig: 
23. Krystalle mit einer zweizähligen Inversionsaxe (Gr.4). Symbol: $J^{2}$. Tetrathionsaures Kalium.

24. Krystalle mit einer vierzähligen Inversionsaxe (Gr.9). Symbol: $J^{4}$. Kein Beispiel.

25. Krystalle mit einer sechszähligen Inversionsaxe (Gr. 19). Symbol: $J^{6}$. Kein Beispiel.

Da jede zweizählige Inversionsaxe $J^{2}$ identisch ist mit einer senkrecht zu ihr stehenden Symmetrie-Ebene (so wie die zweizählige Axe der zusammengesetzten Symmetrie $Z^{2}$ identisch ist mit einem Symmetriecentrum), und da ferner die $J^{6}$ in der Form $L^{3} . J^{2}$ dargestellt werden kann, so erhalt man für 23 und 25 das Vorhandensein einer Symmetrie-Ebone. Die Klasse 24 ist jene merkwürdige Abtheilung, welche in der Groth'schen Ableitung durch $Z^{4}$ charakterisirt wird.

Nun sind die vorangehenden Symmetrieklassen der Reihe nach mit der Inversionsaxe zu combiniren; zunächst sollen dem Symmetrieschema z weizählige Inversionsaxen binzugefügt werden. Indem diese Operation mit den Klassen 2 bis 25 vorgenommen wird, erhält man eine Anzahl neuer Klassen; bei einer anderen Anzahl fuhrt die Combination zu bereits vorhandenen Symmetrieschemen, oder sie erweist sich — wie bei der Abtheilung 8,9 und 11 - als unmöglich und im Widerspruche mit dem Rationalitätsgesetze.

Die neuen Abtbeilungen sind:

26. Fügt man zu einer zweizähligen Symmetrieaxe eine zu ihr senkrechte zweizählige Inversionsaxe, so besitzt das entstehende Polyëder noch eine zweite zweizählige Inversionsaxe, welche zu beiden vorigen senkrecht steht. Man hat also Krystalle mit einer zweizähligen Symmetrieaxe und zwei senkrecht zu derselben stehenden, einander unter $90^{\circ}$ schneidenden zweizähligen Inversionsaxen (Gr. 7). Symbol: $L^{2} \cdot J_{1}{ }^{2} \cdot J_{11}{ }^{2}$. Kieselzinkerz.

27. Durch Combination von 3 mit $J^{2}$ erhält man Krystalle mit einer dreizähligen Symmetrieaxe und drei zweizähligen Inversionaxen senkrecht zu derselben (Gr. 20). Symbol: $L^{3} \cdot 3 J^{2}$. Turmalin.

28. Ebenso aus 4: Krystalle mit einer vierzähligen Symmetrieaxe und zweimal zwei zweizähligen Inversionsaxen senkrecht zu derselben (Gr. 14). Symbol : $L^{4} \cdot 2 J_{1}^{2} \cdot 2 J_{11}{ }^{2}$. Silberfluorid.

28. Ebenso aus 5: Krystalle mit einer sechszähligen Symmetrieaxe und zweimal drei zweizähligen Inversionsaxen senkrecht zu derselben (Gr. 26). Symbol: $L^{6} \cdot 3 J_{1}^{2} \cdot 3 J_{11}{ }^{2}$. Wurtzit.

30. An dem Symmetrieschema der Klasse 6 können zweizählige Inversionsaxen in der Mitte zwischen zwei zweizähligen Symmetrieaxen angebracht werden. Diese letzteren werden dadurch gleich und man erhält Krystalle mit einer zweizähligen Symmetrieaxe, ferner in der Ebene senk- 
recht zu derselben $z$ wei gleiche, zweizählige, sich unter $90^{\circ}$ schneidende Symmetrieaxen und die Winkel dieser halbirend zwei zweizählige Inversionsaxen (Gr. 11). Symbol: $L^{2} \cdot 2 L_{1}{ }^{2} \cdot 2 J_{1}{ }^{2}$. Kupferkies.

31. Aehnlich lassen sich in Klasse 7 zwischen die zweizähligen Symmetrieaxen Inversionsaxen einschalten. Man erhält dadurch Krystalle mit einer dreizähligen Symmetrieaxe, in der Ebene senkrecht zu derselben drei zweizählige Symmetrieaxen und die Winkel der vorigen halbirend drei zweizählige Inversionsaxen (Gr. 22). Symbol: $L^{3} \cdot 3 L^{2} \cdot 3 J_{1}{ }^{2}$. Kein Beispiel.

32. Bei Abtheilung 10 ist die Einschaltung zweizähliger Inversionsaxen zwischen je zwei zweizählige Synmetrieaxen möglich. Man erhält hierdurch Krystalle mit vier dreizähligen und drei zweizăhligen Symmetrieaxen, feíner die Winkel der vorigen halbirend scchs zweizählige Inversionsaxen (Gr. 31). Symbol : $4 L^{3} .3 L^{2} \cdot 6 J^{2}$. Fablerz.

Es ist nicht schwer nachzuweisen, dass jede weitere Combination der Symmetrie-Elemente entweder zu schon abgeleiteten Klassen fuhrt, oder krystallographisch unmöglich ist.

Durch das Vorstehende ist der Nachweis erbracht, dass man die 32 Klassen der Krystalle ableiten kann blos mit Hülfe der Begriffe: Symmetrieaxe, Symmetriecentrum und der Combination beider: Inversionsaxe. Damit glaube ich aber auch den Nachweis erbracht zu haben, dass man, wie es bereits von Bravais geschehen ist, die Begriffe Symmetrie-Ebene und Symmetriecentrum als völlig gleichberechtigt anzusehen habe.

Vergleicht man die hier gegebene Ableitung mit der im Groth'schen Buche, so wird man finden, dass der Vortbeil grösserer Anschaulichkeit und Klarheit bald hier, bald dort zu finden ist. Sonach schiene es als das zweckmässigste, sowohl die Symmetrie-Ebenen, als das Symmetriecentrum zur Ableitung zu verwenden, nachdem in einer Einleitung die Beziebungen zwischen den einzelnen Symmetrie-Elementen dargelegt wurden.

Eine solche Ableitung würde zunächst bis $\mathrm{Nr}, 22 \mathrm{mit}$ der vorliegenden parallel laufen. Hierauf würde die Symmetrie-Ebene eingefuhrt (Nr. 23). Dann würde die Combination der Symmelrie-Ebene senkrecht zu einer einzelnen Symmetrieaxe vorgenommen, welche zu zwei neuen Klassen Nr. 25 (Gr. 19) und Nr. 31 (Gr. 25) gelangen lässt. Dann kăme die Combination von Symmetrie-Ebenen, die sich in einer Symmetrieaxe schneiden; diese führt zu den Klassen Nr. 26, 27, 28, 29, 30, 32 (Gr. 7, 20, 14, 26, 11, 31). Endlich bleibt allein ubrig die tetragonal-bisphenoidische Klasse Nr. 24 (Gr. 9), zu deren Ableitung nothwendiger Weise die zusammengesetzte oder Inversions-Symmetrie herangezogen werden muss. 